\title{
From High- to Super-resolution Mass Spectrometry
}

\author{
Yury O. Tsybin*
}

\begin{abstract}
High-resolution mass spectrometry (MS) is indispensable for the molecular-level analysis of biological and environmental samples with great intra- and inter-molecular complexity. Here, we summarize developments in Fourier transform mass spectrometry (FTMS), the flagship of high-resolution MS techniques, accomplished in our laboratory. Particularly, we describe the recent and envisioned progress in structural analysis of: i) isolated large proteins and their simple mixtures, with a focus on monoclonal antibodies, via top-down, middle-down, and extended bottom-up mass spectrometry; ii) complex protein mixtures and proteomes via extended bottom-up proteomics; and iii) crude oil fractions and similar complex molecular mixtures. Despite the unequivocal success in molecular structural analysis, the demonstrated results clearly indicate that the compromise between MS acquisition speed (throughput) and achievable resolution level inhibits further advances of MS applications in the areas related to life, environmental, and material sciences. To further advance beyond state-of-the-art FTMS capabilities in these areas, we present the technique of super-resolution mass spectrometry that has been pioneered by our laboratory.
\end{abstract}

Keywords: Extended bottom-up proteomics · Filter diagonalization method (FDM) ·

Fourier transform mass spectrometry (FTMS) - Middle-down proteomics · Petroleomics · Resolution .

Super-resolution mass spectrometry · Top-down proteomics

\section{Introduction}

Mass spectrometry (MS) is the most sensitive and specific analytical technique for molecular and macromolecular structural analysis. [1] The rapidly improving MS analytical characteristics aim to match the growing demands of molecular analysis in life, material, and environmental sciences. The current level of MS performance enables speciation of extremely complex mixtures of organic and inorganic molecules needed for environmental, e.g. in MS-based petroleomics and dissolved organic matter analysis, as well as material sciences applications. For life sciences applications, high-performance MS is indispensable for identification and in-depth characterization, including quantitation and modifications mapping, of metabolites and proteins embedded in complex molecular environments, e.g. as realized in MS-

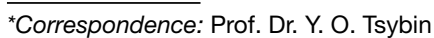
Biomolecular Mass Spectrometry Laboratory Ecole Polytechnique Fédérale de Lausanne EPFL BCH 4307

$\mathrm{CH}-1015$ Lausanne

Tel.: + 41216939751

E-mail: yury.tsybin@epfl.ch based metabolomics and proteomics. [2] Nevertheless, the gap between the state-ofthe-art MS performance and the analytical demands imposed by the complexity of molecular systems of interest has not yet been bridged. ${ }^{[3,4]}$ One of the limiting factors that delays further advancement in MS-based applications is the throughput of analysis in experiments with time constraints. Particularly, high-resolution MS data should be acquired faster and without sacrificing spectral dynamic range. Indeed, sample complexity in modern MS applications requires a certain level of resolution defined by the ability to distinguish isobaric compounds, e.g. species with similar mass-to-charge $(\mathrm{m} / \mathrm{z})$ ratios. From the practical consideration, resolution is typically defined as the ratio of the peak location on the $m / z$ scale to the peak width at the half of the peak maximum (FWHM). The narrower the peak - the higher the resolution. Resolution above 30'000 may be referred to as high resolution, albeit resolution classification depends on the type of a mass spectrometer for which it is defined. Although for most applications the required levels of resolution can now be achieved with high-resolution MS instruments, the throughput of these measurements is not yet sufficient. For example, when Fourier transform mass spectrometry (FTMS, vide infra) is employed, qualitative peptide analysis typically requires resolution of 15-30'000 (50-100 ms ion detection time), whereas protein structural analysis asks for 60-120'000 resolu- tion (200-400 ms ion detection time) and above. Significantly higher demands are imposed on quantitative peptide and protein analysis (e.g. 500'000 resolution and the corresponding $1.5 \mathrm{~s}$ ion detection time in mass defect-based isobaric tags methods), as well as complex molecular mixture analysis (e.g. petroleomics, vide infra) and peptide isotopic fine structure analysis (demands exceed 1'000'000 resolution and $3 \mathrm{~s}$ ion detection time). As a result, other MS analytical characteristics, e.g. mass accuracy, dynamic range and sensitivity, suffer from the compromise between speed and resolution. Furthermore, although very high, the ultimate levels of resolution achieved in the MS experiments without time constraints (e.g. petroleomics) remain limited, with a few exceptions. ${ }^{[5]}$ Therefore, increasing the overall resolution level may open new horizons in molecular structure analysis, as well as provide insights into the related fundamental molecular physics and chemistry. ${ }^{[6]}$

Herein, we describe an ongoing effort undertaken at the Biomolecular Mass Spectrometry Laboratory (LSMB) at Ecole Polytechnique Fédérale de Lausanne (EPFL) to accelerate state-of-the-art highresolution FTMS and to develop superresolution mass spectrometry (SRMS) that addresses the above described limitations to advance MS-based applications in life, material, and environmental sciences. To achieve these advances, innovations in the allied fields of signal processing, fundamental ion physics, and analytical instru- 
mentation are required. Finally, following the motto "to break the rules you must first master them" of a famous Swiss watchmaker we report our achievements in the corresponding fields of targeted molecular analysis, proteomics, and petroleomics obtained with the state-of-the-art FTMS technology.

\section{Fourier Transform Mass Spectrometry: The Starting Point}

Fourier transform mass spectrometry (FTMS) provides superior analytical characteristics, specifically resolution and mass accuracy, to all other MS techniques. ${ }^{[7-10]}$ Additionally, FTMS uniquely offers an intriguing capability for further improvement through advances in the generation and treatment of time-domain ion signals, commonly referred to as transients. The two widely employed FTMS instruments are those based on an ion cyclotron resonance (ICR) and an orbitrap ion trap (or cells). The first operates in a homogenous magnetic field environment, whereas the second is an electrostatic field-based mass analyzer.[11] The third, emerging type of FTMS mass analyzer is an electrostatic ion trap employing a multi-reflection time-offlight (TOF) mass analyzer. ${ }^{[12-14]}$ The main competitors of FTMS instruments are the state-of-the-art reflectron TOF MS with single and multiple turns of ions in their flight path between an ion source and the ion collision-based detector. They provide up to $50^{\prime} 000$ resolution at $1000 \mathrm{~m} / \mathrm{z}$ with $10-100 \mathrm{~Hz}$ scan rate. Note, spectral dynamic range is a function of the scan rate in TOF MS and thus accumulation of a high quality mass spectrum (dynamic range above 3 orders of magnitude) is time-consuming. Recent implementation of highresolution (up to 10 bit on the vertical, amplitude, scale) and high-frequency digitizers has significantly increased the achievable dynamic range of TOF MS. Some of the modern ion detectors employ the power of charge sensitive devices for ion detection in TOF MS, which demonstrates improved performance specifically for heavy ion detection, particularly important for intact protein level mass analysis. [15]

The underlying common principle of mass analysis in FTMS instruments is the generation and detection of periodic ion motion. The measured transients can be Fourier transformed to yield the corresponding frequency spectra. Application of the known relations between the frequencies of ion oscillations and their $\mathrm{m} / \mathrm{z}$ values enables generation of the mass spectra. The described standard procedure of FTMS mass spectra generation works most efficiently when ion signal components in transients are sinusoi- dal. Deviations from sinusoidal nature of transient components lead to formation of additional features in the Fourier spectra, e.g. high-order frequency harmonics and sidebands around the peaks of interest, which may be manifested as artifacts in mass spectra. ${ }^{[16]}$ The presence of these components can be minimized by the rational design of the mass analyzers that aim for generation and analysis of the principal harmonic or principal frequency multiple. Typically, electrodes for induced current detection in FTMS are made sufficiently large to integrate ion motion over a given path. For instance, in FT-ICR MS, most ICR cells feature a pair of $90^{\circ}$ electrodes for ion detection and work most efficiently at the first harmonic or first frequency multiple ion detection.

On the other hand, the application of FT signal processing to the sinusoidal signals in modern FTMS results in a limitation on the resolution level achieved in a given time period of ion signal detection (FT uncertainty principle).[17] The FT resolution limitation in modern FTMS is at the level of the achieved resolution equal to about one unit per 2-4 periods of ion oscillation. Therefore, to achieve a resolution of 100'000 the recorded transient should contain a substantial number (200'000400'000) of ion oscillation periods. The provided estimation of resolution per period is based on the transient of a sufficient length, required for determination of ion oscillation frequency. A key question is: How should the FT resolution limitation achieved with modern FTMS instruments best be overcome to accelerate the acquisition speed of high-resolution MS data? The approach advocated by our laboratory is comprised of both MS hardware (mass analyzers) and software (signal processing) innovations, the synergy of which should result in development of super-resolution mass spectrometry (SRMS), vide infra. Before the SRMS description we will present some of the currently most promising

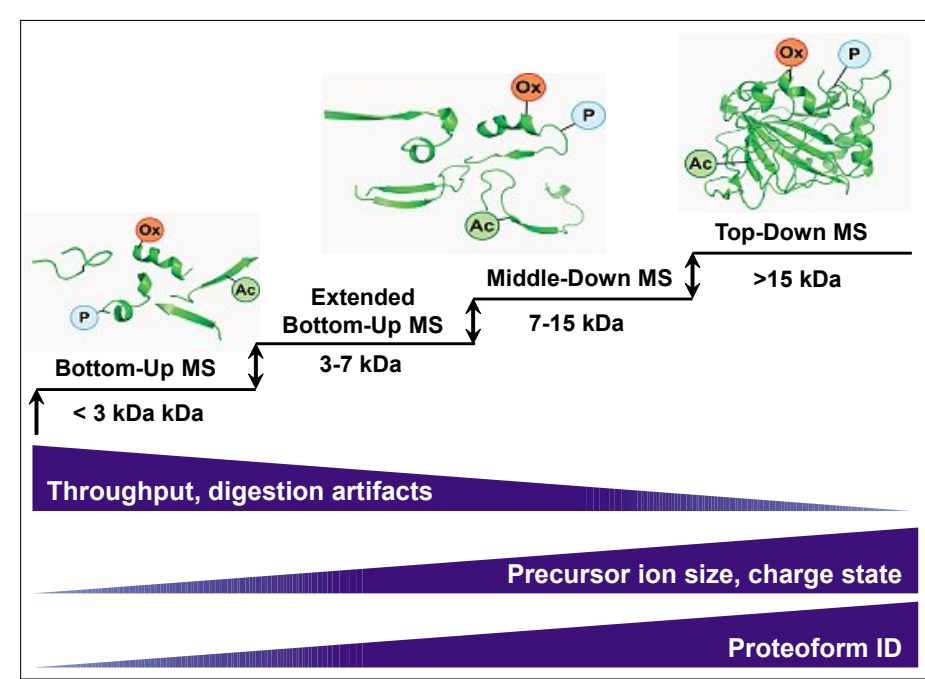

FTMS application areas that demonstrate the need for SRMS development.

\section{Advancing FTMS-based Applications for Molecular Structure Analysis}

Analytical characteristics of FTMS allow for its application not only for the analysis of molecular mixtures separated in solution or gas phase prior to injection into the mass analyzer, but also for the direct analysis of extremely complex molecular mixtures. However, the performance of both these approaches requires further improvement. Below we summarize our recent advances in methods and techniques enabling progress of these FTMS applications.

\section{Proteome Analysis: Extended Bottom-Up Proteomics}

The standard method of complex biological mixtures, e.g. proteomes, analysis by MS is through bottom-up proteomics (Fig. 1). The power of this approach lies in the excellent analytical characteristics of MS and liquid chromatography (LC) for analysis of isolated short (10-30 amino acids in length) peptides produced via well-established enzymatic digestion protocols. However, the extremely high number of components and more than 10 orders of magnitude dynamic range of protein concentrations in the real-life biological samples significantly reduce the currently achieved depth of the MS analysis. Here, increased throughput of the high-resolution MS analysis potentially offered by SRMS would be of great interest.

On the other hand, already provided by commercial instruments, LC-based separation and high-resolution MS capabilities enable efficient and rapid mass analysis of longer (30-70 amino acids) peptides. We have recently suggested referring to the proteome analysis based on identification
Fig. 1. An overview of mass spectrometrybased proteomics strategies. 
of these long peptides as extended bottomup proteomics (Fig. 1). ${ }^{[18,19]}$ Increasing the size of enzymatically derived peptides effectively reduces the total number of peptides produced by each protein, which may significantly reduce the complexity of peptide mixtures submitted for LC-MS analysis and increase confidence in protein identification with longer peptides (higher protein sequence coverage provided by a single peptide). Application of SRMS methodology may further increase the throughput of MS analysis, which necessarily should be a high-resolution one, to accommodate the needs for long peptide ${ }^{13} \mathrm{C}$-isotopic-level analysis. The remaining bottleneck is how best to produce these long peptides.

It is known that restricted by digestion time proteolysis with typically employed enzymes, such as trypsin, produces long peptides. However, the reproducibility and substrate specificity of these reactions have not yet been sufficiently studied, whereas preliminary studies demonstrate fundamental limitations of this approach. Thus, we first attempted to answer the following questions: what should be the specificity of a protease for optimum production of 3-7 kDa peptides? And what proteases of enzymatic or chemical nature exist that would match the determined optimum specificity rules? To address the first question we performed an extensive bioinformatics investigation, which demonstrated that a specific cleavage at a given amino acid or a doublet of amino acids will not produce a sufficient number of 3-7 $\mathrm{kDa}$ unique peptides to represent the complete proteome. ${ }^{118]}$ On the other hand, combination of cleavages at rare amino acids, e.g. Trp or Met, or doublets of amino acids, e.g. dibasic sites, with a limited tendency toward non-specific cleavages may indeed provide the required pools of peptides. In our laboratory, we implemented and characterized two workflows for production of long peptides: i) a workflow based on digestion with in-house expressed and purified enzyme, secreted aspartic protease Sap9:[19] and ii) a workflow based on proteolytic reactions with chemicals, e.g. $\mathrm{CNBr}{ }^{[20]}$ Interestingly, despite a large number of studies described in the prior literature on protein digestion with chemical reagents, there have been no reported attempts of their application for protein mixture analysis. Both workflows demonstrated intriguing capabilities for extending bottom-up proteomics to the analysis of longer peptides.

The specificity of Sap9 enzyme to the dibasic sites, as originally suggested in the literature, has been only partially confirmed by our large-scale studies.[19] However, combining dibasic specificity with unspecific cleavages enabled efficient and rapid ( $1 \mathrm{~h}$ digestion reaction) production of 3-7 $\mathrm{kDa}$ peptides. A particular advantage of Sap9 compared with other enzymes is its comparable-level activity in a wide range of $\mathrm{pH}$ values. It is known that enzymatic digestion under $\mathrm{pH}$ conditions typical for trypsin, e.g. $\mathrm{pH} 7-8$, may induce protein artifacts, such as deamidation, whereas performing digestion at $\mathrm{pH}$ $\sim 5$ reduces the presence of such artifacts. These characteristics of Sap9 performance are particularly beneficial for the targeted protein structural analysis, e.g. of monoclonal antibodies, immunoglobulins $\mathrm{G}$ (IgGs). On the other hand, the non-specific nature of Sap9 reduces the efficiency of its application to large-scale proteomics studies. Therefore, further optimization of the experimental parameters is required.

Further increasing size, above $7 \mathrm{kDa}$, of proteolytically-derived peptides requires significant modification of LC, MS, and MS/MS parameters to deliver optimum performance. MS-based approach that targets analysis of such peptides, or more correctly said - proteins (polypeptides above $5 \mathrm{kDa}$ are typically referred to as proteins), is termed middle-down mass spectrometry (proteomics) (Fig. 1). From the analytical (MS) perspective, the middle-down approach overlaps with the top-down one, as described below.

\section{Protein Analysis: Top-Down and Middle-Down Proteomics}

Top-down MS or proteomics refers to tandem mass spectrometry (MS/MS)based analysis of intact proteins or protein complexes, as well as their large, typically $>15 \mathrm{kDa}$, subunits (Fig. 1). The complexity of MS and MS/MS data obtained during analysis of such large molecules dictates the need for high-resolution and high mass accuracy MS performance. ${ }^{[21,22]}$ Theoretically, the top-down approach is the ultimate goal in MS-based protein and proteome analysis - as it allows for proteoform-level molecular analysis. ${ }^{[23]}$ Already at the MS level information on a protein sample may be employed to quantitatively describe proteoform heterogeneity, but only when the mass difference between the proteoforms is sufficiently large. In our work, we employed this MS-level approach for monitoring glycosylation profiles following stable and transient monoclonal antibody production and storage. ${ }^{[24]}$ Avoiding the enzymatic or chemical digestion step minimizes the introduction of possible artifacts during sample preparation, e.g. deamidation, and reveals the connectivity of protein complex subgroups and modifications. ${ }^{[25,26]}$ Adding a MS/MS step to structural analysis of proteins and protein complexes potentially provides protein primary structure information (sequence and location of modifications) and protein complex stoichiometry. For example, MS/MS of intact IgGs and their large subunits is of a particular importance for their quality control and for improving the drug discovery process. With these incentives in mind, we extended the range of MS/MS applications to $150 \mathrm{kDa}$ intact monoclonal antibodies structural analysis, first on a high-resolution time-of-flight (TOF) MS and then on a standard and high-field Orbitrap FTMS (Fig. 2). ${ }^{[21,22]}$ By applying electron transfer dissociation (ETD) for MS/MS of intact protein ions, we not only achieved protein sequence coverage exceeding previous (limited) attempts of top-down collision induced dissociation (CID) MS/MS-based analysis of monoclonal antibodies, but also increased the molecular weight of proteins fragmented with ETD MS/MS in general. [27] These results have further contributed to the already significant interest in the MSbased structural analysis of monoclonal antibodies. In follow-up work, Marshall and co-workers achieved similar sequence coverage of monoclonal antibodies with electron capture dissociation (ECD) on an FT-ICR MS and demonstrated isotopic-level resolution of antibodies.[28,29] Brodbelt and co-workers matched these results by advancing high-resolution Orbitrap FTMS analysis of antibodies by achieving their isotopic-level resolution ${ }^{[30]}$ and attempting to fragment their intact ions with a promising method of $193 \mathrm{~nm}$ ultraviolet photo-dissociation (UVPD). ${ }^{[31]}$

Despite a certain level of success achieved with top-down MS of monoclonal antibodies, the obtained sequence coverage remained at the $33 \%$ limit and small size modifications, e.g. oxidation, could not be efficiently identified with this approach. To address these issues, we reduced the size of antibodies by their site-specific and artifact-free digestion in a hinge region with a dedicated enzyme, IdeS (Fig. 2). [25] Depending on the extent of the additional reduction of disulfide bonds the resulting antibody subunits could be of $100 \mathrm{kDa}$, $50 \mathrm{kDa}$, or $25 \mathrm{kDa}$. Application of ETD and higher collisional energy (HCD) MS/ MS to these large fragments on high-field Orbitrap FTMS resulted in increased sequence coverage (up to 70\%) and improved modifications analysis, including the unambiguous assignment of oxidation sites. Importantly, analysis of 25-50 kDa instead of $150 \mathrm{kDa}$ proteins simplifies the related LC-MS/MS experiment and substantially increases the performance for the analysis of antibody mixtures. Due to the involvement of an enzymatic digestion step to the procedure, the described analysis can be referred to as middle-down approach, whereas the size of proteins, $25-100 \mathrm{kDa}$, justifies classification of this approach as top-down. 


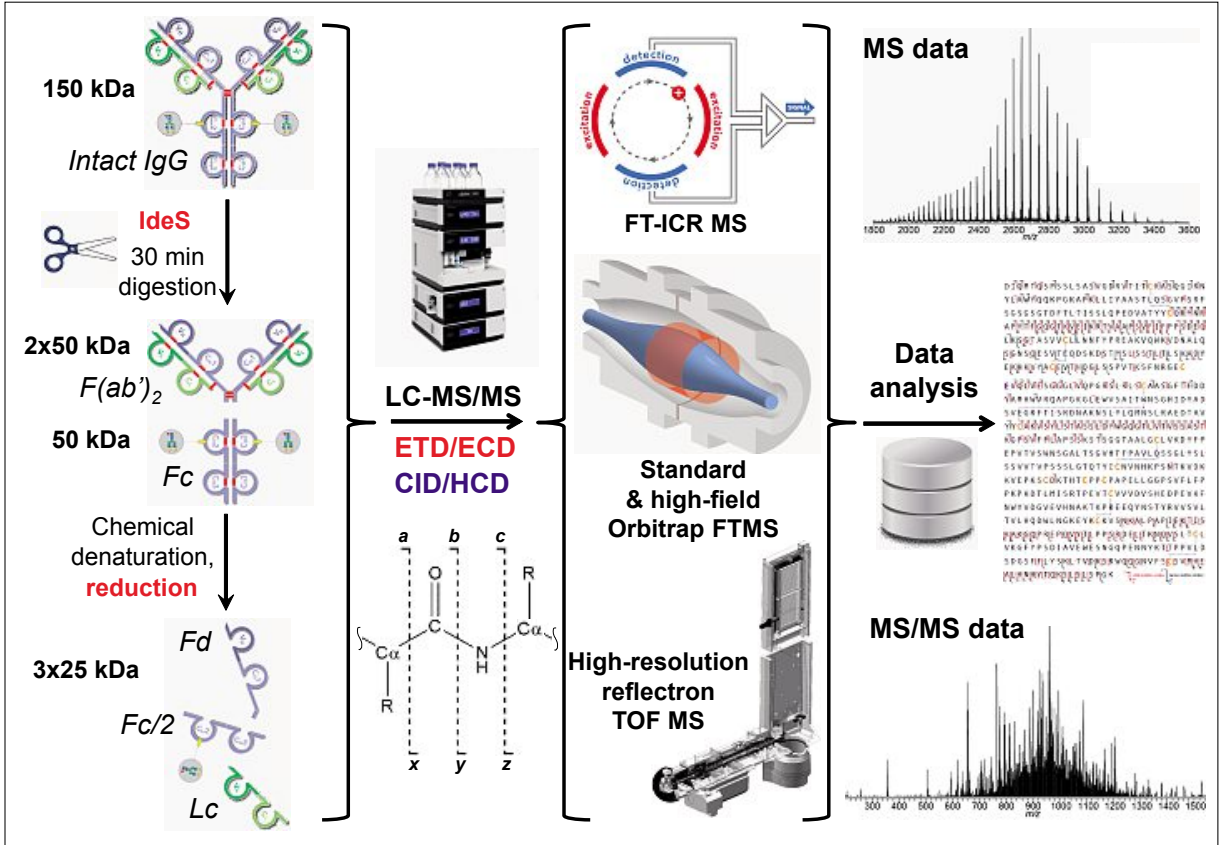

Fig. 2. High-resolution mass spectrometry: top-down and middle-down mass spectrometry workflows.

In addition to analysis of monoclonal antibodies, in different collaborations we have shown the utility of the top-down MS approach in the analysis of proteindrug complexes and clinical studies. In collaboration with Dyson and co-workers, we investigated the binding of anti-cancer organometallic drugs and drug candidates to small proteins, which revealed the insights of the mode of action of these small molecule drugs. ${ }^{[32-34]}$ In collaboration with Hochstrasser and co-workers, we applied top-down MS to increase the robustness and speed of the amino-acid level modifications analysis of clinically relevant proteins, starting with hemoglobin and related hemaglobinopathies diagnostics. ${ }^{[35]}$

\section{Complex Mixture Analysis: Petroleomics}

Petroleomics generally refers to direct infusion high-resolution mass spectrometry of extremely complex molecular mixtures. ${ }^{[36,37]}$ Most often MS-based petroleomics deals with crude oil and its fractions. The allied application areas cover environmental sciences (including analysis of dissolved organic matter from water reservoirs) and material sciences (including reaction products of high complexity, e.g. those obtained upon high-pressure decomposition and hydrogenation of fullerenes, ${ }^{[38]}$ as well as biofuels analysis). Efficient separation of these complex molecular mixtures by liquid or gas chromatography is often not feasible, making high-resolution MS analysis indispensable. Recent advances in gas-phase ion separation technique, ion mobility (IM) time-of-flight (TOF) MS, led to substantial progress of this complementary analytical
FT-ICR MS-based petroleomics, such as mass spectra recalibration procedures, to Orbitrap FTMS is not possible due to the differences in fundamentals of ion motion between these two FTMS technologies. Therefore, the main steps in the LSMB workflow include custom-developed algorithms that consider the particular characteristics of Orbitrap FTMS data: i) generation and acquisition of transients with extended lengths, up to 3-6 s. Transient data treatment in absorption mode FT spectral representation is particularly useful, thus the use of the eFT algorithm is encouraged;[40] ii) thresholding of raw mass spectral data by distinguishing analyte and noise distributions via plotting peak component density against the decimal logarithm of peak intensity; ${ }^{[41]}$ iii) iterative recalibration of mass spectral data with an empirical estimation of the mass calibration function, which takes into account not only the $m / z$ values of the peaks but also their abundances; ${ }^{[40]}$ iv) the obtained mass accuracy values allow unambiguous peak assignment and v) data visualization following the hexagonal class representation method based on relative abundance versus compound classes plot.[16] The described workflow has been successfully applied to high-field Orbitrap FTMS-based analysis of crude oil fractions of low to medium complexity. ${ }^{[39]}$ Notably, FTMS transients of only $1.5 \mathrm{~s}$ duration were employed in this pioneering study. Increased sample complexity requires measurement of higher resolution FTMS data, which is now readily offered by the acquisition of longer, up to 3-6 s, transients. ${ }^{[42]}$ Nevertheless, further improvements in resolution performance are required to increase the through-
(Fig. 3).[39] Interestingly, direct application of data analysis strategies developed for method that is particularly useful once the Until recently, only high-resolution T-ICR MS was capable of delivering required for petrolion and mass accuracy have developed an alternative workflow, based on a high-field Orbitrap FTMS, that delivers petroleomics-grade performance for many related samples without the need for an expensive super-conducting magnet

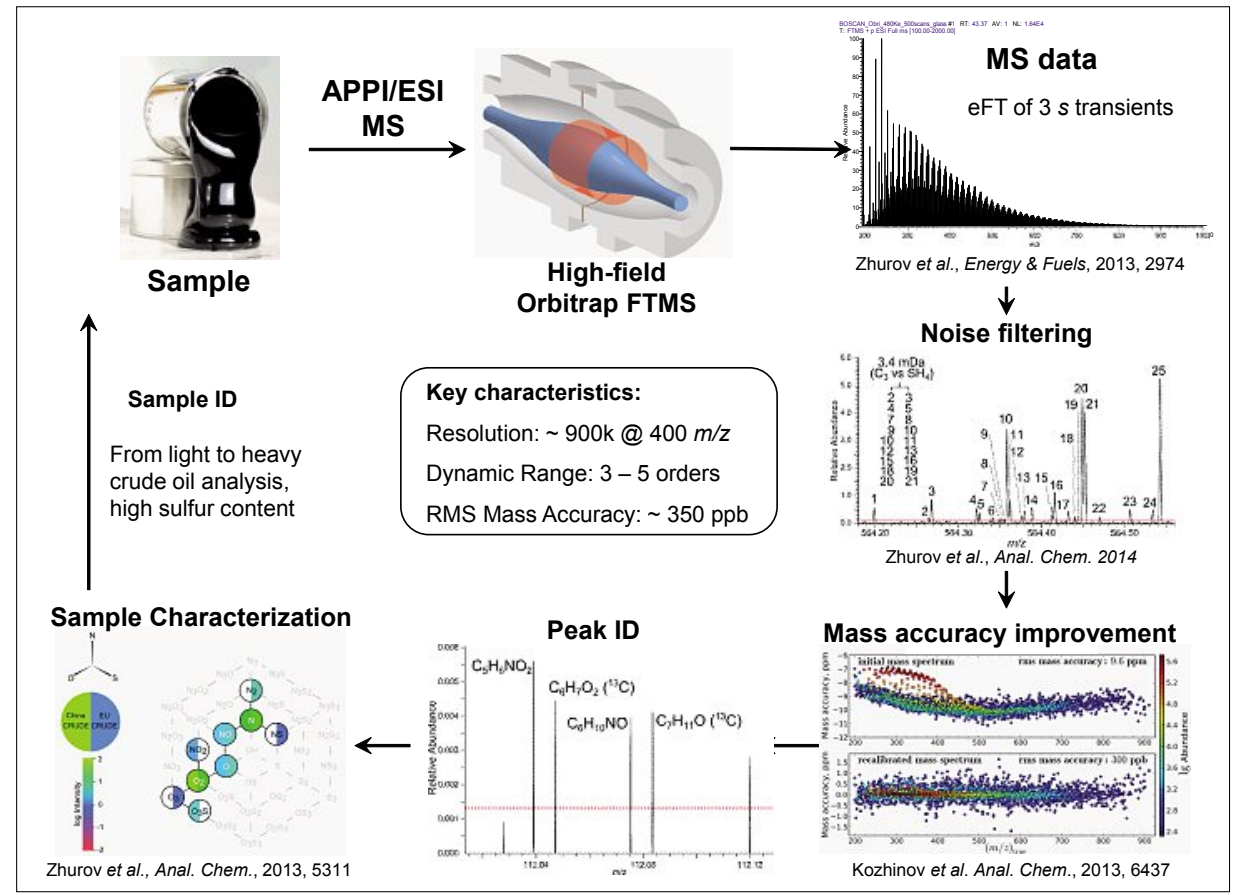

Fig. 3. High-resolution mass spectrometry: Orbitrap FTMS-based petroleomics workflow. 
put of petroleomics experiments and for analysis of heavy (above $1000 \mathrm{Da}$ ) isobaric ions demonstrating less than $1 \mathrm{mDa}$ mass difference. These advances would be welcome not only for Orbitrap but also for ICR FTMS-based petroleomics.

\section{Super-resolution Mass Spectrometry: Pushing the Limits of FTMS Applications}

Above, we have presented results in petroleomics as well as top-down, middle-down, and extended bottom-up proteomics demonstrating the substantial advances achieved in molecular analysis with modern high-resolution MS. Nevertheless, extremely high sample complexity often exceeds performance of FTMS-based platforms employed in these approaches. Super-resolution mass spectrometry (SRMS) is aimed to advance FTMS by delivering high-resolution MS data faster (at least 10 times). By our definition, SRMS is an analytical technique that employs superresolution methods of signal processing for transient time-to-frequency conversion. ${ }^{[43]}$ Therefore, SRMS may surpass FTMS in terms of resolution as the quantitative form of time-to-frequency uncertainty principle of super-resolution methods of signal processing is less strict compared to that of the FT-based signal processing. The current roadmap for SRMS development at LSMB (Fig. 4) is based on the synergy of: i) innovations in MS instrumentation, with a focus on generation of transients composed of user-defined functions, e.g. harmonic frequency multiples; and ii) advances in signal processing, with a focus on super-resolution signal processing method implementation; ${ }^{[43]}$ supported by iii) highperformance data acquisition systems development for maximizing the information output and matching the requirements of signal processing algorithms. On the other hand, the MS datasets generated following the presented workflows (Figs 2 and 3 ), especially petroleomics mass spectra, being extremely complex in $m / z$ and abundance directions, are particularly useful for SRMS development and evaluation. In the following we briefly summarize our main achievements in SRMS development.

\section{Instrumentation for Advanced FTMS and Super-resolution Mass Spectrometry}

A standard approach of accelerating FTMS is to increase the frequency of harmonic components in the FTMS transients. That is typically achieved by increasing the magnetic field strength in FT-ICR MS or electrostatic field strength in Orbitrap FTMS. However, there are opportunities for increasing the detected frequency with-

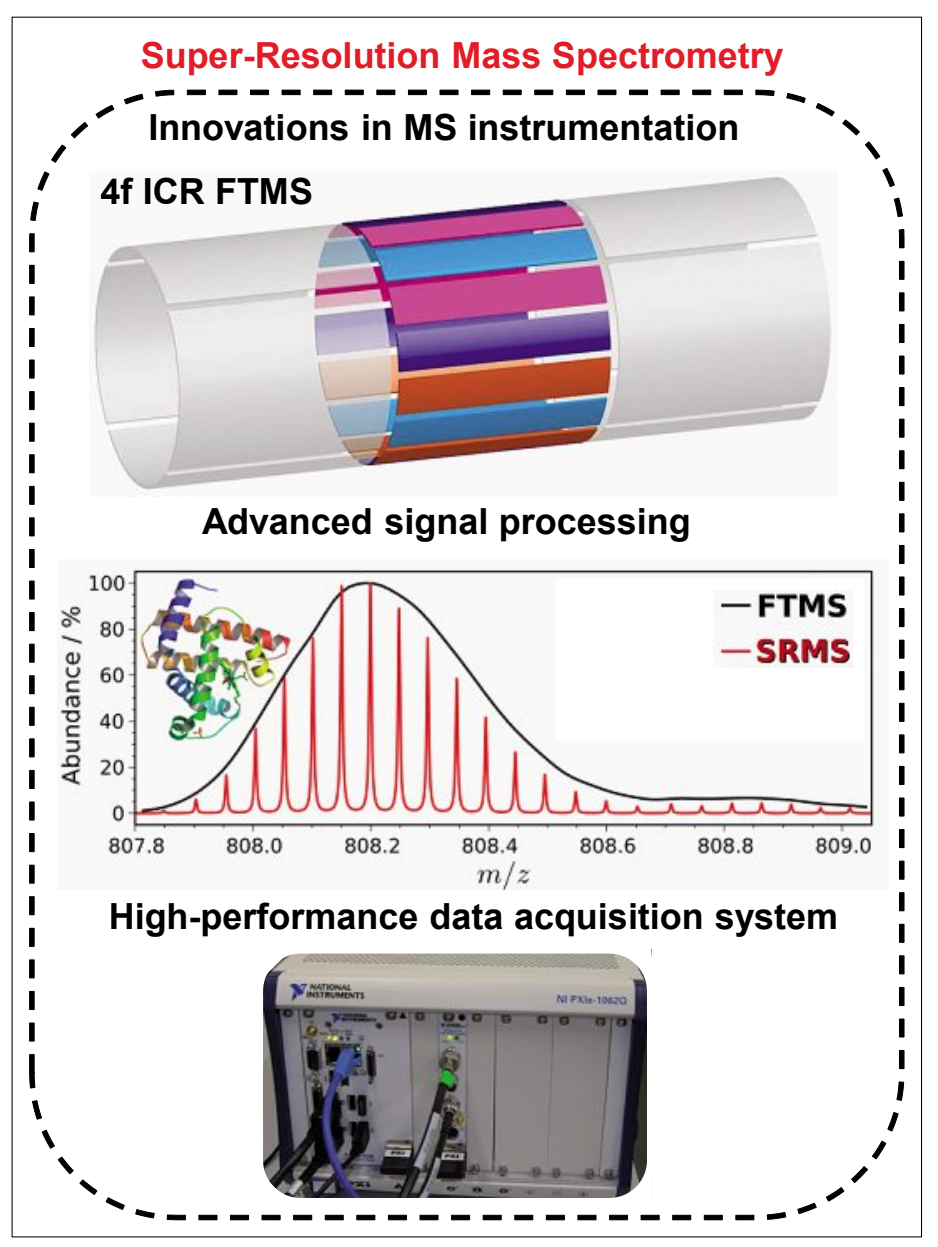

Fig. 4. Superresolution mass spectrometry: a synergy of innovations in MS instrumentation, advanced signal processing, and data acquisition.

out changing the magnetic or electric field strength. For example, we demonstrated that doubling the frequency (and, thus, the resolution) on a commercial FT-ICR MS is feasible without significant hardware modifications. ${ }^{[44]}$ Direct detection of higher-order harmonics or frequency multiples has been discussed in the literature, ${ }^{[45]}$ but a dedicated implementation on a modern FTMS instrument suitable for biomolecular analysis has been missing. Recently, we implemented the 16-electrode ICR cell on the proteomics-grade $10 \mathrm{~T}$ FT-ICR MS (LTQ FTMS) platform that enables the efficient recording of the quadruple frequency multiple (Fig. 4, top panel). ${ }^{[46]}$ This implementation accelerates acquisition of high-resolution FTMS data by four times, providing the expected benefits for molecular and macromolecular structure analysis. Can we go beyond this achievement to further increase the recorded frequency?

To address the objectives discussed above, we invented and implemented a dedicated ICR cell with modified trapping, excitation, and space-charge fields. ${ }^{[47]}$ The preliminary data demonstrates the envisioned capabilities of this ICR cell for faster high-resolution MS, as well as for improved analytical characteristics of standard-speed high-resolution FTMS. The experimental data were obtained with the in-house-built high-performance data acquisition system (National Instruments electronics based), enabling a high (up to $100 \mathrm{MHz}$ ) frequency sampling rate at substantial vertical resolution (16 bit) and rapid data transfer for acquisition of transients of any length (Fig. 4, bottom panel). The matching signal processing should be developed to maximize the advantages provided by this ICR cell. Our preliminary results in the corresponding signal processing development are briefly summarized below.

\section{Super-resolution Mass Spectrometry Signal Processing}

At the software level, SRMS development involves implementation of non-FT and advanced FT methods of signal processing for transient analysis and goes in two ways: i) signal processing of harmonic transients with methods that allow the magnitude-mode FT resolution limitation to be overcome; and ii) signal processing of transients composed of userdefined functions. To advance the former way, we implemented two super-resolution methods of signal processing, the welldeveloped parameter estimators, filter diagonalization method (FDM) ${ }^{[43,48]}$ and a least squares fitting (LSF) ${ }^{[49]}$ to analyze the experimental ICR and Orbitrap FTMS transients. Fundamentally, the super-resolution methods, including FDM and LSF, 
should allow peaks in mass (frequency) spectra to be resolved using shorter transients than required by FT. Indeed, our data (including the success in implementation of ICR cells providing high frequency multiples and harmonics) demonstrates that due to a discrepancy between the FT requirements (FT uncertainty principle) on the length of the transient signal and the instrumentally achieved coherence of ion motion in the phase space, there is room for possible resolution improvement. Our results with FDM and LSF application to experimental ICR and Orbitrap FTMS data demonstrate that the gain in resolution performance is governed by spectral irregularity. We reported experimental data where the gain ranges from a minimum (2-fold) (Fig. 4, middle panel), to significant (up to 30 -fold) values, e.g. as in the analysis of isotopic fine structures of peptides, ${ }^{[48]}$ and in protein quantitation with isotopicallycoded isobaric tags with mass defects. To advance the latter way, the efficient signal processing methods are still to be developed. Importantly, their development for SRMS may find utility in other areas of science and technology facing similar challenges.

\section{Conclusions and Outlook}

High-resolution MS continues to increase its role in molecular structural analysis. Already accepted as an important analytical technique by life and environmental sciences researchers, it has begun to demonstrate its importance and utility for clinical and medical applications. Advances in instrumentation and signal processing, including those summarized here, open new avenues for improved molecular analysis, e.g. top-down mass spectrometry of isolated intact monoclonal antibodies, protein-drug complexes, or clinically relevant proteins for their proteoform-level analysis; middle-down and extended bottom-up proteomics of complex protein mixtures for improved qualitative and quantitative protein analysis; and petroleomics for a complete speciation of extremely complex mixtures of small molecules. For instance, the methods developed in our laboratory for biotherapeutics, monoclonal antibodies, structural analysis can significantly reduce the time and artifacts induced by sample preparation in classical methods which makes them useful for antibodybased drug development and characterization. These methods can also be extended to characterization of antibody-drug conjugates, which are a new generation antibody-based therapeutics.

Super-resolution mass spectrometry, implemented at LSMB for real-life FTMS data, makes its first steps through funda- mentals development and implementation as an original and complementary analytical technique to FTMS. Importantly, preliminary results validate the feasibility of this approach, whereas the FTMS applications, including those demonstrated here, confirm the need for faster high-resolution MS. Further development of advanced signal processing and high-performance data acquisition techniques coupled with MS instrumentation shall lead to a robust and routine SRMS technology. On the other hand, exceptional computational power requirements for transient data analysis limit more rapid development and acceptance of the SRMS technology. The allied FTMS/ SRMS instrumentation development program at our laboratory led to implementation of the quadruple frequency multiple ICR cell and innovation of a novel type of ICR cells that demonstrate superior analytical performance to the currently employed ICR mass analyzers.

In addition to the advances mentioned above, during its first eight years LSMB has also contributed to the physical chemistry side of tandem mass spectrometry. ${ }^{[27]}$ Particularly, we revealed new insights into electron capture and transfer dissociation fundamentals. For instance, we discovered and performed mechanistic studies of the periodic distribution of ECD/ETD product ions from peptides exhibiting a tendency to form an alpha-helical secondary structure; [50-53] completed knowledge on the role of amino acid side-chains in the ECD/ ETD of peptides with beta amino acids in the sequence; ${ }^{[54]}$ suggested and computationally supported a mechanism of heterolytic N-C backbone cleavage in ECD/ ETD. ${ }^{[55]}$ In parallel, we applied improved understanding of ECD/ETD fundamentals for peptide structure analysis: created a method for distinguishing N-terminal and C-terminal product ions for improved peptide sequencing; ${ }^{[56]}$ investigated the mode of action of tissue transglutaminase on peptide deamidation ${ }^{[26]}$ and its possible implication in Alzheimer-related beta-amyloid peptide aggregation kinetics. ${ }^{[57]}$ Improving the understanding of fundamentals behind molecular interaction with electrons, photons, and other molecules is indispensable for revealing the molecular structure-activity-function triad. Therefore, physical chemistry naturally complements the synergy of analytical and bio-chemistry for improved biomolecular structure analysis that constitutes the heart of LSMB interdisciplinary research program.

\section{Acknowledgements}

Former and current LSMB group members and collaborators are warmly acknowledged for all the exciting and productive interactions over the eight years of laboratory life, since its inception to the current time. We thank Thermo
Scientific for enabling additional features to our instruments and overall technical support. We are grateful to Bruker Daltonics for supporting our top-down mass spectrometry development initiatives with TOF MS. The work was supported by the Swiss National Science Foundation (Projects 200021-125147, 200021147006, and 128357), the European Research Council (ERC Starting grant 280271), and EPFL.

Received: February 13, 2014

[1] E. Sabido, N. Selevsek, R. Aebersold, Curr. Opin. Biotechnol. 2012, 23, 591.

[2] Y. O. Tsybin, L. Fornelli, A. N. Kozhinov, A. Vorobyev, S. M. Miladinovic, Chimia 2011, 65, 641.

[3] R. A. Zubarev, Proteomics 2013, 13, 723.

[4] A. Michalski, J. Cox, M. Mann, J. Proteome Res. 2011, 10, 1785.

[5] I. A. Boldin, E. N. Nikolaev, Rapid Commun. Mass Spectrom. 2011, 25, 122.

[6] A. G. Marshall, C. L. Hendrickson, S. D. H. Shi, Anal. Chem. 2002, 74, 252A.

[7] A. G. Marshall, C. L. Hendrickson, Ann. Rev. Anal. Chem. 2008, 1, 579.

[8] F. Xian, C. L. Hendrickson, A. G. Marshall, Anal. Chem. 2012, 84, 708 .

[9] R. A. Zubarev, A. Makarov, Anal. Chem. 2013, $85,5288$.

[10] M. Scigelova, M. Hornshaw, A. Giannakopulos, A. Makarov, Mol. Cell. Proteomics 2011, 10, M111.009431.

[11] A. Makarov, Anal. Chem. 2000, 72, 1156.

[12] M. Gonin, US Patent 10/449328, 2003.

[13] A. Verenchikov, Patent GB20100000649, 2010.

[14] D. Zajfman, Y. Rudich, I. Sagi, D. Strasser, D. W. Savin, S. Goldberg, M. Rappaport, O. Heber, Int. J. Mass Spectrom. 2003, 229, 55.

[15] J. H. Jungmann, L. MacAleese, J. Visser, M. J. J. Vrakking, R. M. A. Heeren, Anal. Chem. 2011, 83, 7888.

[16] K. O. Zhurov, A. N. Kozhinov, Y. O. Tsybin, Anal. Chem. 2013, 85, 5311.

[17] A. N. Kozhinov, T. Aushev, Y. O. Tsybin, in Proc. 61 $1^{\text {st }}$ ASMS Conference on Mass Spectrometry and Allied Topics, Minneapolis, MN, USA, 2013

[18] Ü. A. Laskay, A. A. Lobas, K. Srzentic, M. V. Gorshkov, Y. O. Tsybin, J. Proteome Res. 2013, 12, 5558.

[19] U. Laskay, A., K. Srzentic, L. Fornelli, O. Upir, A. N. Kozhinov, M. Monod, Y. O. Tsybin, Chimia 2013, 67, 244.

[20] K. Srzentic, G. Karateev, L. Fornelli, L. Levitsky, A. A. Lobas, U. A. Laskay, M. V. Gorshkov, D. Ayoub, E. Dubikovskaya, Y. O. Tsybin, in Proc. $62^{\text {nd }}$ ASMS Conference on Mass Spectrometry and Allied Topics, Baltimore, USA, 2014.

[21] Y. O. Tsybin, L. Fornelli, C. Stoermer, M. Luebeck, J. Parra, S. Nallet, F. M. Wurm, R. Hartmer, Anal. Chem. 2011, 83, 8919.

[22] L. Fornelli, E. Damoc, P. M. Thomas, N. L. Kelleher, K. Aizikov, E. Denisov, A. Makarov, Y. O. Tsybin, Mol. Cell. Proteomics 2012, 11, 1758.

[23] L. M. Smith, N. L. Kelleher, Consortium TopDown Proteomics, Nature Methods 2013, 10, 186.

[24] S. Nallet, L. Fornelli, S. Schmitt, J. Parra, L. Baldi, Y. O. Tsybin, F. M. Wurm, New Biotechnol. 2012, 29, 471.

[25] L. Fornelli, D. Ayoub, K. Aizikov, A. Beck, Y. O. Tsybin, Anal. Chem. 2014, DOI: 10.1021/ ac4036857.

[26] L. Fornelli, A. W. Schmid, L. Grasso, H. Vogel, Y. O. Tsybin, Chem. Eur. J. 2011, 17, 486.

[27] K. O. Zhurov, L. Fornelli, M. D. Wodrich, U. A. Laskay, Y. O. Tsybin, Chem. Soci. Rev. 2013, 42,5014 . 
[28] S. G. Valeja, N. K. Kaiser, F. Xian, C. L. Hendrickson, J. C. Rouse, A. G. Marshall, Anal. Chem. 2011, 83, 8391.

[29] Y. Mao, S. G. Valeja, J. C. Rouse, C. L. Hendrickson, A. G. Marshall, Anal. Chem. 2013, 85, 4239.

[30] J. B. Shaw, J. S. Brodbelt, Anal. Chem. 2013, $85,8313$.

[31] J. B. Shaw, W. Li, D. D. Holden, Y. Zhang, J. Griep-Raming, R. T. Fellers, B. P. Early, P. M. Thomas, N. L. Kelleher, J. S. Brodbelt, J. Am. Chem. Soc. 2013, 135, 12646.

[32] S. M. Meier, Y. O. Tsybin, P. J. Dyson, B. K. Keppler, C. G. Hartinger, Anal. Bioanal. Chem. 2012, 402, 2655.

[33] F. Mendes, M. Groessl, A. A. Nazarov, Y. O. Tsybin, G. Sava, I. Santos, P. J. Dyson, A. Casini, J. Med. Chem. 2011, 54, 2196.

[34] C. G. Hartinger, Y. O. Tsybin, J. Fuchser, P. J. Dyson, Inorg. Chem. 2008, 47, 17.

[35] D. C. Graca, P. Lescuyer, L. Clerici, Y. O. Tsybin, R. Hartmer, M. Meyer, K. Samii, D. F. Hochstrasser, A. Scherl, J. Am. Soc. Mass Spectrom. 2012, 23, 1750.

[36] A. G. Marshall, R. P. Rodgers, Proc. Nat. Acad. Sci. USA 2008, 105, 18090

[37] C.-S. Hsu, C. L. Hendrickson, R. P. Rodgers, A. M. McKenna, A. G. Marshall, J. Mass Spectrom. 2011, 46, 337.
[38] S. M. Luzan, Y. O. Tsybin, A. V. Talyzin, J. Phys. Chem. C 2011, 115, 11484.

[39] K. O. Zhurov, A. N. Kozhinov, Y. O. Tsybin, Energy \& Fuels 2013, 27, 2974.

[40] A. N. Kozhinov, K. O. Zhurov, Y. O. Tsybin, Anal. Chem. 2013, 85, 6437.

[41] K. O. Zhurov, A. N. Kozhinov, L. Fornelli, Y. O. Tsybin, Anal. Chem. 2014, DOI: 10.1021/ ac403278t.

[42] E. Denisov, E. Damoc, O. Lange, A. Makarov, Int. J. Mass Spectrom. 2012, 325, 80.

[43] A. N. Kozhinov, Y. O. Tsybin, Anal. Chem. 2012, 84,2850

[44] A. Vorobyev, M. V. Gorshkov, Y. O. Tsybin, Int. J. Mass Spectrom. 2011, 306, 5.

[45] E. N. Nikolaev, M. V. Gorshkov, A. V. Mordehai, V. L. Talrose, Rapid Comm. Mass Spectrom. 1990, 4, 144.

[46] K. O. Nagornov, M. V. Gorshkov, A. N. Kozhinov, Y. O. Tsybin, in Proc. $11^{\text {th }}$ European FTMS Workshop, Paris, France, 2014.

[47] K. O. Nagornov, A. N. Kozhinov, Y. O. Tsybin, in Proc. 62 ${ }^{\text {nd }}$ ASMS Conference on Mass Spectrometry and Allied Topics, Baltimore, MD, USA, 2014
[48] S. M. Miladinovic, A. N. Kozhinov, M. V. Gorshkov, Y. O. Tsybin, Anal. Chem. 2012, 84, 4042 .

[49] T. Aushev, A. N. Kozhinov, Y. O. Tsybin, J. Am. Soc. Mass Spectrom. 2014, submitted.

[50] H. Ben Hamidane, A. Vorobyev, Y. O. Tsybin, Eur. J. Mass Spectrom. 2011, 17, 321.

[51] H. Ben Hamidane, D. Chiappe, R. Hartmer, A. Vorobyev, M. Moniatte, Y. O. Tsybin, J. Am. Soc. Mass Spectrom. 2009, $20,567$.

[52] A. Vorobyev, H. Ben Hamidane, Y. O. Tsybin, J. Am. Soc. Mass Spectrom. 2009, 20, 2273.

[53] V. Pouthier, Y. O. Tsybin, J. Chem. Phys. 2008, 129, 095106.

[54] H. Ben Hamidane, A. Vorobyev, M. Larregola, A. Lukaszuk, D. Tourwe, S. Lavielle, P. Karoyan, Y. O. Tsybin, Chem. Eur. J. 2010, 16, 4612 .

[55] M. D. Wodrich, K. O. Zhurov, A. Vorobyev, H. Ben Hamidane, C. Corminboeuf, Y. O. Tsybin, J. Phys. Chem. B 2012, 116, 10807.

[56] Y. O. Tsybin, H. He, M. R. Emmett, C. L. Hendrickson, A. G. Marshall, Anal. Chem. 2007, 79, 7596.

[57] A. W. Schmid, E. Condemi, G. Tuchscherer, D. Chiappe, M. Mutter, H. Vogel, M. Moniatte, Y. O. Tsybin, J. Bio. Chem. 2011, 286, 12172. 